\title{
EL DERECHO ADMINISTRATIVO ESPAÑOL ANTE LAS NEUROCIENCIAS Y EL NEURODERECHO: DESARROLLOS Y PERSPECTIVAS ${ }^{1}$
}

\section{SPANISH ADMINISTRATIVE LAW IN LIGHT OF NEUROSCIENCES AND NEUROLAW: DEVELOPMENTS AND PERSPECTIVES}

\author{
Carlos-Alberto AMOEDO-SOUTO
}

Resumen: El presente trabajo pretende aproximar el lector o lectora al impacto, presente y futuro, de las neurociencias en el ámbito del Derecho público. Como ha ocurrido en otras revoluciones científicas, el conocimiento neurocientífico disponible está generando nuevas realidades cuya regulación jurídica es ya inaplazable. En el ámbito internacional se ha consolidado ya una nueva disciplina conocida como Neuroderecho ("neurolaw”). Sin embargo, en España permanecemos todavía al margen de estos desarrollos, por lo que el trabajo defiende la necesidad de una mayor atención de la comunidad jurídica a los mismos.

\begin{abstract}
This paper addresses the present and future impacts of neurosciences on public law. Just as previously raised by other scientific revolutions, neuroscientific insights are creating new legal realities that law must manage sooner or later. A global legal approach is coming under the name of "neurolaw". However, these kind of features have already no development in our legal tradition. That's why we stand up for paying more attention to these issues.
\end{abstract}

Palabras clave: Neurociencias, neuroderecho, regulación, España

Key words: neurosciences, neurolaw, regulation, Spain

\footnotetext{
${ }^{1}$ El presente trabajo tiene su origen en la comunicación presentada al Seminario de Teoría y Método sobre neurociencias y Derecho Administrativo, celebrado en Santiago de Compostela el 25 de mayo de 2018. El Seminario de Teoría y Método es una red de excelencia coordinada por las Universidades Autónoma de Madrid y Valencia, y subvencionada por el Ministerio de Economía, referencia DER201681747-REDT.
} 


\section{Ecos de los avances neurocientíficos en la ciencia jurídica española}

Las relaciones entre neurociencias y derecho - entre el conocimiento disponible en materia de neurociencia y las disciplinas jurídicas - constituyen una temática relativamente nueva en nuestro país.

Aunque en el ámbito internacional se habla desde por lo menos quince años de los problemas que los constantes avances del conocimiento de nuestra arquitectura cerebral -su hardware y su software, si se quiere- plantean para aquellas disciplinas jurídicas más cercanas a la regulación de la conducta humana, en nuestro país apenas las dogmáticas penalista y procesalista han desarrollado interesantes debates sobre las consecuencias del conocimiento neurocientífico disponible en sus conceptos y esquemas metodológicos propios.

En particular, la evidencia neurocientífica disponible ha puesto de manifiesto que cuando pretendemos tomar decisiones, nuestro cerebro desencadena una serie de patrones de percepciones y conexiones neuronales más o menos automáticos, que condicionan y limitan de manera decisiva nuestro (presunto) albedrío: el mito civilista de la autonomía de la voluntad.

Nuestras respuestas conductuales, tanto en las patologías mentales (adicciones, trastornos de la personalidad, neurosis, psicosis, etc.) como en el funcionamiento "normal" del cerebro, están ampliamente regladas por procesos cerebrales "automáticos". De esta manera, el encadenamiento del sujeto a su subconsciente que había sido propugnado por el psicoanálisis durante el siglo $\mathrm{XX}$, ha sido superado en nuestros días por otro encadenamiento, esta vez empíricamente sustentado, a las bases neurobiológicas de funcionamiento del cerebro ${ }^{2}$.

Estas ideas han provocado una especie de latigazo sobre algunas de las instituciones centrales de la dogmática penal.

Fundamentalmente, se cuestiona la existencia de libre albedrío individual sobre la toma de decisiones, con la colateral posibilidad misma de reprochabilidad subjetiva del comportamiento delictivo (culpabilidad) ${ }^{3}$, la erosión de los fundamentos de la

\footnotetext{
${ }^{2}$ Para un estado del arte sobre esta materia, vid. los capítulos de C. Summerfield y A. Blangero, "Perceptual decision-making: what do we know and what do we not know", y G. UgAZIO y C. RufF, "Neural control of social decisions: causal evidence from brain stimulation studies", ambos incluídos Jean-Claude Dreher y Léon Tremblay (eds.), Decision Neuroscience, Londres, Academic Press, 2017, pp. 149-160, y 233-142, respectivamente.

${ }^{3}$ En España, quizá quepa atribuir el mayor mérito a Eduardo DEMETRIO CRESPO, catedrático de derecho penal de la Universidad de Castilla La Mancha e investigador principal del proyecto "Neurociencias y Derecho Penal", financiado por el Ministerio de Innovación y Ciencia. Este proyecto ha animado la discusión transdisciplinar e interuniversitaria española, y cuyos resultados pueden leerse en Eduardo Demetrio Crespo (dir.) y Manuel Maroto Calatayud (coord.), Neurociencias y derecho penal: nuevas perspectivas en el ámbito de la culpabilidad y tratamiento jurídico-penal de la peligrosidad, Madrid, Edisofer, 2013. Ha abordado también estas perspectivas la monografía dirigida por Bernardo José FeIJoo SÁnchez, Derecho Penal de la culpabilidad y neurociencias, Civitas Thomson Reuters,
} 
prevención especial y de la viabilidad de la reeducación del delincuente mediante la combinación de privación de libertad y terapéutica conductual.

De ahí que, por ejemplo, una de las consecuencias colaterales de las neurociencias haya sido el refuerzo empírico de los fundamentos del positivismo criminológico y de las políticas criminales de inocuización: dado que el delincuente es incorregible, digámoslo así, por la propia arquitectura de su "sistema operativo" cerebral, la resocialización como función de la pena deviene inútil: un riesgo inasumible para la sociedad ${ }^{4}$; de tal modo que la única política criminal pertinente para evitar la reiteración delictiva consiste en la reclusión o imposición al reo de medidas de seguridad permanentes. Con ello, asistimos a una relectura securitaria $-\mathrm{y}$ tecnológicamente avanzada- de planteamientos propios del positivismo criminológico-penal que ya parecían haberse superado, y que confluyen de manera muy alarmante con el denominado por Günther Jakobs como Derecho Penal del Enemigo.

Por su parte, los procesalistas han comenzado a debatir sobre las repercusiones de las investigaciones sobre marcadores biológicos de estados neurales, fundamentalmente para evaluar la viabilidad de su utilización como prueba pericial en el proceso: los detectores de mentiras o polígrafos, incluídos los basados en técnicas de resonancia magnética funcional, ya son válidamente utilizados en algunos estados norteamericanos y en países como India. Más allá del delicado tema de la probatio procesal de la verdad o la mentira, la neurociencia está en trance de abrir otros campos probatorios sobre la subjetividad humana ${ }^{5}$. Tal es el caso de la objetivación o medición del dolor, o la mejora del diagnóstico de enfermedades mentales.

\section{El escaso eco en la doctrina administrativista y la apertura del ventanuco de las ciencias del comportamiento}

En el ámbito del Derecho administrativo, sin embargo, el interés suscitado por los avances neurocientíficos ha sido escaso.

Hasta el momento, no ha repercutido ni sobre temas dogmáticos más próximos al ámbito penal -como es la elaboración del tema de la culpabilidad en el ámbito sancionador, al calor de la teoría de la unidad del Ius Puniendi estatal- o al ámbito procesal - como es el uso de pericias sustentadas en nuevas técnicas neurocientíficas en

2012. Posteriormente, Eduardo Demetrio Crespo ha colectado diversos artículos en la monografía Neurociencias y Derecho Penal, Buenos Aires-Montevideo, editorial B de F, 2017. A mi juicio, en este debate merece mención especial la monografía de José Antonio RAMOS VÁzQUEZ, Ciencia, libertad y Derecho penal (aporías del determinismo y defensa de la libertad de acción como base del sistema penal, Valencia, Tirant Lo Blanch, 2013.

${ }^{4}$ Cfr. Jesús María SiLva SÁncheZ, "El retorno de la inocuización. El caso de las reacciones jurídico penales frente a los delincuentes sexuales violentos", en Luis Alberto ARROYO ZAPATERO e Ignacio Berdugo Gómez de la Torre (coords.). Homenaje al Doctor Marino Barbero Santos: in memoriam, Ediciones de la Universidad de Salamanca, Cuenca, 2001, pp. 699-710.

5 Sobre ello, vid. Michele TARUfFo y Jordi Nieva Fenoll (dirs.), Neurociencia y proceso judicial, Madrid, Marcial Pons, 2013. 
el ámbito contencioso-administrativo-, ni tampoco sobre el núcleo duro de sus objetos o metodologías.

Nuestra disciplina ha desarrollado poca reflexión sobre la subjetividad de nuestros sujetos clásicos; el desarrollo de una mirada especializada sobre el derecho de esa persona jurídica abstracta y sin alma que es la Administración desde los enfoques del positivismo normativo nos ha hecho descuidar la visión del administrado o el funcionario en cuanto sujetos destinatarios de la norma jurídico-administrativa que, a su vez, reelaboran subjetivamente el mandato o mandatos en ella contenidos conforme a múltiples preferencias y elecciones a veces no explícitas. En buena medida, permanecemos anclados todavía en una precomprensión decimonónica de estos sujetos destinatarios de las normas administrativas como hombres adultos, racionales, propietarios y buenos padres de familia, siendo más bien reciente la atención a los menores, a las mujeres, a los discapacitados y a los pobres como sujetos administrados.

Salvando a Alejandro Nieto - estoy pensando en su El arbitrio judicial, publicado en 2000-, no hemos problematizado excesivamente el papel de la subjetividad y la "arracionalidad" (sesgos cognitivos, ideológicos, emocionalidad) de los operadores jurídico-administrativos, o el análisis de la voluntad en el derecho administrativo. Baste con recordar a este propósito el escaso desarrollo de la culpabilidad en el ámbito sancionador, o del reconocimiento de circunstancias psíquicas que puedan eximir o atenuar la responsabilidad en las Leyes reguladoras de la potestad sancionadora y disciplinaria ${ }^{6}$.

Es cierto, con todo, que la introducción contemporánea de referencias positivas al grado de culpabilidad y la intencionalidad como criterios legales de fijación normativa e imposición individualizada de las sanciones $^{7}$ ha contribuído a abrir el debate en el plano jurisprudencial, pues "el elemento subjetivo de la infracción, en la medida en que se encuentra en un ámbito interno que ordinariamente no puede ser objeto de prueba directa, solo puede acreditarse a través de su inferencia a partir de los datos objetivos debidamente acreditados" $"$. Lo cual marca una interesante tendencia a la subjetivación de un derecho, el sancionador administrativo, tradicionalmente más inclinado hacia la configuración de su responsabilidad propia como "inobservancia" objetiva de la norma prohibitiva, ahora reinterpretada como omisión de la diligencia debida en el cumplimiento de la norma de cuidado. Estamos lejos, sin embargo, de incorporar herramientas que nos permitan individualizar y adaptar la subsunción de la norma

\footnotetext{
${ }^{6}$ Apenas la Ley vasca 2/1998, de 20 de febrero, de la potestad sancionadora de las administraciones públicas de la Comunidad Autónoma Vasca, desarrolla con detalle en sus artículos 6 y 8 la previsión de aplicación "con matices" de las causas de exención o atenuación de la responsabilidad que se prevén en el Código Penal, dejando la puerta abierta a otras causas específicas que se establezcan en las normas sancionadoras sectoriales.

${ }^{7}$ Vid. los artículos 130 y 131 de la Ley 30/1992, ya derogada, y los preceptos homólogos de la ley 40/2015, de régimen jurídico del sector público (28 y 29).

${ }^{8}$ Cfr. STS sala $4^{\text {a }}$ de 7 de febrero de 2017, RJ 20171933. De ahí la copiosa jurisprudencia sobre sanciones tributarias nacida alrededor de la configuración de la "diligencia necesaria en el cumplimiento de las obligaciones tributarias" como causa específica de exención de la responsabilidad prevista por el artículo 179.2.d) de la Ley General Tributaria.
} 
administrativa a las circunstancias particulares - en especial, a las mentales - que guían el comportamiento del "sujeto administrado" o incluso del "sujeto administrador".

Hasta el momento, la apertura de la doctrina administrativista al mundo de las neurociencias contemporáneas se está operando a través de un estrecho ventanuco: el de las behavioral sciences.

Las behavioral sciences agrupan a un conglomerado de tradiciones científicas que han confluído sobre el estudio empírico de la conducta humana mediante la hibridación con la psicología, y entre las que cabe mencionar las behavioral economics, las behavioral policies y una buena parte de la ciencia política norteamericana del siglo XX.

Tal confluencia nos permite comprender mejor las bases empíricas de la conducta y de la toma humana de decisiones. Al iluminar el contenido de la caja negra de la interacción social a un nivel micro, incluyendo el amplio papel que juegan los procesos inconscientes, las ciencias de la conducta proporcionan herramientas con las que mejorar el grado de eficacia de las políticas públicas. Esto es, los instrumentos gerenciales de orientación de la conducta individual y agregada de los ciudadanos hacia la consecución del interés general.

En España, ha sido el doctor Juli Ponce Solé (Universidad de Barcelona), quien con más insistencia ha divulgado entre la doctrina administrativista española las aportaciones que durante los últimos diez años la perspectiva gerencialista behaviorista ha desarrollado con éxito en todo el mundo bajo el concepto de nudging ${ }^{10}$.

\footnotetext{
${ }^{9}$ Piénsese, por ejemplo, en las dificultades - cabe añadir: insuperables - que hay en el terreno de la función pública para evaluar la aptitud mental del personal al servicio de las administraciones públicas y, en su caso, solicitar la incapacidad para el servicio. No sólo vivimos de espaldas a las enfermedades mentales; en materia de acreditación de capacidades para el acceso a la función pública, en plena época informática y de adaptación de nuestros planes de estudio universitarios al Espacio Europeo de Educación Superior, sorprende que conservemos unos procesos selectivos -especialmente los de acceso a los grandes cuerpos jurídicos- cuya principal habilidad mental evaluada sea la capacidad memorística. No menos sorprendente es que estemos asistiendo a la implantación de unas variantes de la "subjetividad administrativa" de tanto éxito como dudosa consistencia científica: el mindfulness y el coaching. Recientemente, la Escuela Gallega de Administración Pública ha ofertado sendos cursos de este estilo dirigidos al personal de las administraciones gallegas. Vid. Resolución de 20 de abril de 2018, por el que se convoca curso monográfico sobre Mindfulness productivo (DOG n ${ }^{\circ} 80$, de 25 de abril), y la ficha del curso "coaching, la herramienta para el cambio".

${ }^{10}$ Cfr. Juli PONCE SOLÉ, "Nudging, simplificación procedimental y buen gobierno regulatorio. El Derecho Administrativo del siglo XXI y sus relaciones con las ciencias sociales", en Luis MígueZ MACHO, Marcos Almeida CerReda y Diana SANTIAgo Iglesias, La simplificación de los procedimientos administrativos: actas del IX Congreso de la Asociación Española de Profesores de Derecho Administrativo, Santiago de Compostela, EGAP, 2014, pp. 191-216; con el profesor Agustí CERRILlo MARTíNEZ, Innovación en el ámbito del buen gobierno regulatorio: ciencias del comportamiento, transparencia y prevención de la corrupción, INAP, Madrid, 2017. Sobre el nudging son imprescindibles las referencias al libro del premio Nobel Richard THALER y Cass Sunstein, Un pequeño empujón (nugdge), Madrid, Taurus, 2009. Sobre el denominado paternalismo libertario, vid. Cass SunSTEIN, Paternalismo libertario, Barcelona, Herder, 2017. Este mismo autor se ha ocupado de los problemas
} 
En él se incluyen distintas modalidades de "empujoncitos" públicos (desde recordatorios periódicos del vencimiento de plazos para trámites, premios y menciones, estímulos publicitarios como "el tabaco mata" de las cajetillas de tabaco, información como el coste de un tratamiento, reglas por defecto, etc.) que aprovechan el conocimiento neurocientífico disponible para influir en la conducta individual del sujeto administrado, sin llegar a imponer un mandato o prohibición en sentido estricto. De ahí que también se haya cualificado el nudging como de "paternalismo libertario".

Habla por sí solo de su impacto social el hecho de que Estados Unidos, Inglaterra, la OCDE o la Unión Europea hayan incorporado ya esta perspectiva a sus respectivos policy making.

En 2010, el gobierno británico de David Cameron creó el Behavioural Insights Team, con David Halpern a su cabeza, para aplicar las ciencias del comportamiento a las políticas públicas británicas. Sus variados resultados prácticos pueden verse en el informe correspondiente a los años 2016-2017, consultable aquí.

En Estados Unidos, el Presidente Obama firmó el 18 de enero de 2011 una primera Orden Ejecutiva ("Improving regulation and regulatory review") que incluía en su párrafo cuarto el siguiente principio regulatorio para las políticas públicas: "Where relevant, feasible, and consistent with regulatory objectives, and to the extent permitted by law, each agency shall identify and consider regulatory approaches that reduce burdens and maintain flexibility and freedom of choice for the public. These approaches include warnings, appropriate default rules, and disclosure requirements as well as provision of information to the public in a form that is clear and intelligible". E1 15 de septiembre de 2015, firmó la Orden Ejecutiva "Using behavioral science insights to better serve the American people", consultable aquí. La unidad denominada White House Social and Behavioral Science Team ha funcionado hasta la llegada de Donald Trump a la presidencia norteamericana en enero de 2017.

En Francia, desde 2013 la Secretaría General para la Modernización de la Acción Pública y la Dirección General de Hacienda Pública han multiplicado la utilización de los nudges.

En la Unión Europea, el Comité Económico y Social aprobó el 15 de diciembre de 2016 un Dictamen sobre la integración de los nudges (sic) en las políticas europeas ${ }^{11}$. La Comisión Europea ha elaborado también el Behavioral insights applied to Policy. European Report 2016, disponible aquí. 


\section{De las ciencias del comportamiento a las neurociencias: un cambio en el foco de análisis}

Sin embargo, más allá del indudable interés que provoca la aplicación de las ciencias del comportamiento al intervencionismo público y de sus correlativas consecuencias sobre su formalización jurídica "nacional", la neurociencia contemporánea plantea un amplio conjunto de nuevos desafíos a los que el Derecho público y administrativo no van a permanecer ajenos. De hecho, ya no lo están, ni pueden estarlo.

La diferencia de objeto y método entre las ciencias del comportamiento y las neurociencias contemporáneas puede ejemplificarse sencillamente mediante el recurso a uno de los experimentos fundacionales del behaviorismo.

La famosa caja de la paloma fue ideada por uno de los padres fundadores de las ciencias del comportamiento, B.F. Skinner. Por ello, recibe también el nombre de la "caja de Skinner".

Este experimento de condicionamiento del comportamiento mediante el uso del refuerzo (estímulos positivos o negativos) fue utilizado en 1940, en el marco de la II Guerra Mundial, para diseñar el denominado "pingeon project": una paloma entrenada mediante condicionamiento de su comportamiento para "seguir" con sus picoteos sobre un cristal un objetivo móvil y de esta manera orientar hacia ese objetivo una bomba planeadora. Aunque este proyecto demostró ser operativo, fue descartado por el Estado mayor del Ejército de los Estados Unidos en 1944, aunque recuperado por la Armada en 1948.

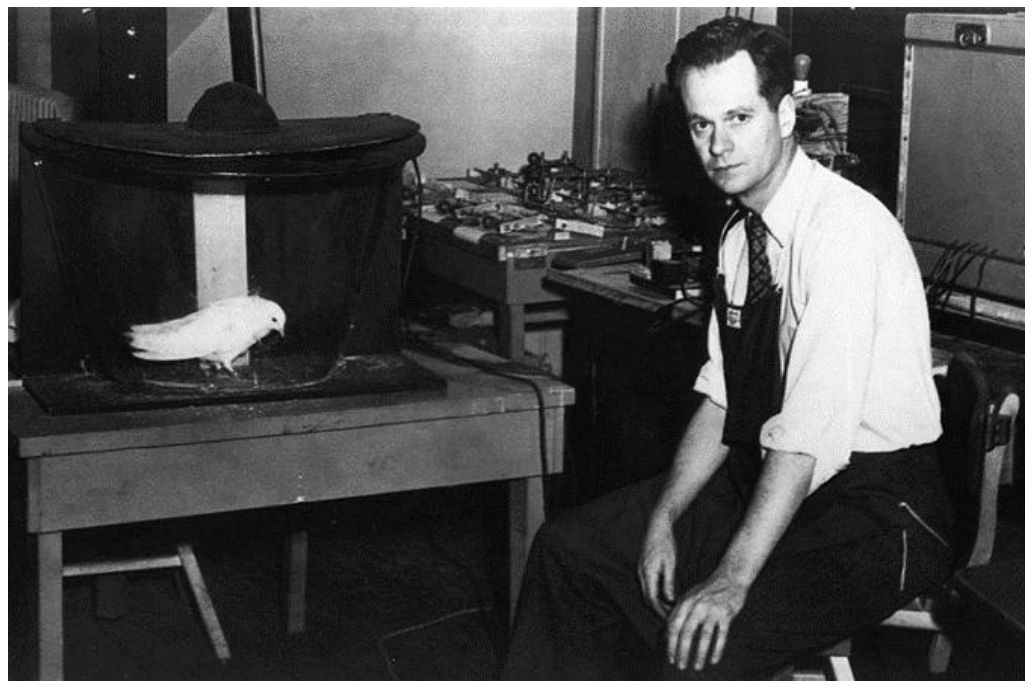

El 29 de marzo de 2018, la Agencia surcoreana de investigación en defensa ha patentado un sistema para controlar el comportamiento de animales con propósitos militares o humanitarios (rescates en zonas devastadas) mediante neuromodulación de las zonas cerebrales de recompensa y miedo: se trata de una aplicación más del conocimiento neurocientífico acumulado en los últimos veinte años sobre el cerebro, que ya no consiste en el aprendizaje condicionado del animal; ya no se trata de la manipulación "psicológica" de su comportamiento mediante estímulos positivos o negativos, sino en la manipulación misma de su cerebro. 


\section{La conquista del cerebro, una nueva revolución científica geoestratégica}

El actual desarrollo de técnicas como la resonancia magnética cerebral, la RMN funcional, la computación neural, el big data o la nanotecnología, está arrojando continuos avances en el estudio de la fisiología de los procesos neuronales, la visualización de la actividad cerebral y el mapa de procesos o funciones cerebrales.

La convergencia, organizada y masiva, de estas tecnologías está produciendo una singular venero de conocimiento científico unificado sobre el órgano humano más misterioso: el cerebro.

Tal convergencia de saberes y técnicas sobre este particular objeto ha generado una nueva carrera científica internacional alrededor del mapa cerebral, la descripción del conectoma (el mapa tridimensional de los entre tres y cuatro trillones de conexiones neuronales que configuran nuestro cerebro) y los procesos neuroquímicos y eléctricos que pautan su funcionamiento. Si los años cuarenta fueron los años de la carrera científica por el dominio de la energía nuclear y los años ochenta los años de la carrera científica por el desentrañamiento de la secuencia del genoma humano, en la actualidad se libra la carrera científica por el acceso al "código fuente" del cerebro humano"

Nuestra actual capacidad de acceso a las bases de los procesos y funciones cerebrales supone un avance cualitativo muy importante respecto de las tradicionales ciencias del comportamiento. Lo que se plantea ahora es poder acceder directamente a la monitorización y control de la actividad cerebral; no sólo "interpretarla" desde el exterior mediante el análisis inductivo de la conducta, o manipularla con premios y castigos, o mediante la administración de psicofármacos.

Esta carrera está siendo alentada desde los gobiernos y las grandes empresas multinacionales del sector TIC. La variaga gama de potentes intereses van desde el desarrollo de aplicaciones con fines militares -mejora de la capacidad de aprendizaje y combate del soldado, contrainteligencia, brain-computer interfaces destinados al combate, marcadores biológicos de estados neurales ${ }^{13}$-, nuevas técnicas terapéuticas de diagnóstico y tratamiento de la salud (neuroestimulación, prótesis sensoriales, nootrópicos ${ }^{14}$ ), hasta una vasta gama de aplicaciones comerciales ${ }^{15}$. Los tratamientos terapéuticos y farmacológicos que el cerebro está en trance de brindarnos constituyen un campo de enorme beneficio social y comercial hacia un futuro muy próximo.

\footnotetext{
${ }^{12}$ Cfr. Nita FARAhANY, "3 reasons brain science is terrific and terrifying", World Economic Forum, 21 enero de 2016, disponible aquí.

${ }^{13}$ Cfr. Opportunities in Neuroscience for Future Army Applications. National Research Council (US), Committee on Opportunities in Neuroscience for Future Army Applications. Washington (DC): National Academies Press (US), 2009. Su descripción puede verse aquí.

${ }^{14}$ Se trata de los fármacos o sustancias capaces de estimular las funciones cerebrales cognitivas.

${ }^{15}$ Recuérdese que, de conformidad con el artículo 21 del protocolo adicional del Convenio de Oviedo, "el cuerpo humano y sus partes, como tales, no deberán ser objeto de lucro o de ventajas comparables".
} 
El cerebro es también el futuro de la informática: alrededor de él, de su arquitectura biológica, química y eléctrica, se está construyendo la neuroinformática, con ordenadores que tratan de replicar "la tecnología" del funcionamiento cerebral que lo convierte en el más eficiente y rápido procesador de información que existe.

Pero dada la ambivalencia de los usos de la ciencia contemporánea, los malos usos de las neurociencias son también potencialmente devastadores.

Recientemente, el proyecto coreano de construcción de un "Terminator" han hecho saltar las alarmas internacionales, poniendo sobre la mesa la insuficiencia de las aproximaciones meramente éticas a estas cuestiones.

El 9 de enero de 2018 Microsoft patentaba una tecnología brain-computer interface para cambiar el estado de una aplicación informática mediante "el pensamiento" (dicho más precisamente: mediante la decodificación de una serie de datos neurológicos captados por una máquina) ${ }^{16}$. Las invenciones y patentes sobre el cerebro se han incrementado exponencialmente en los últimos diez años, y esto no ha hecho más que empezar.

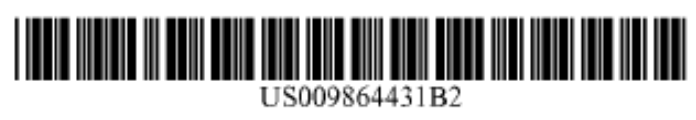
(12) United States Patent
(10) Patent No.:
US $9,864,431 \mathrm{~B} 2$ Keskin et al.
(45) Date of Patent:
Jan. 9, 2018
(54) CHANGING AN APPLICATION STATE USING NEUROLOGICAL DATA
(71) Applicant: MICROSOFT TECHNOLOGY LICENSING, LLC, Redmond, WA (US)
(72) Inventors: Cem Keskin, Seattle, WA (US); David Kim, Bellevue, WA (US); Bill Chau, Sammamish, WA (US); Jaeyoun Kim. Issaquah, WA (US); Kazuhito Koishida, Redmond, WA (US); Khuram Shahid, Seattle, WA (US)
(73) Assignee: Microsoft Technology Licensing, LLC, Redmond, WA (US)

El 2 de abril del año 2013, el presidente Obama presentó la inicativa BRAIN, "Brain Research through Advancing Innovative Neurotechnologies". Con ella, el Instituto Nacional de Salud de los Estados Unidos lanzó un programa para financiar durante 12 años la investigación neurocientífica básica y aplicada, que busca integrar nuevas herramientas tecnológicas y conceptuales con las que desentrañar cómo los patrones dinámicos de la actividad neural son transformados en congnición, percepción, emoción y acción, tanto en los cerebros sanos como en los cerebros patológicos o enfermos.

16 Puede verse también la patente rusa de un sistema de neurocomputación (WO2018080336 (A1), publicada el 3 de mayo de 2018) consistente en controlar dispositivos electrónicos mediante interface cerebro-ordenador. 
En palabras del presidente Obama, "there is this enormous mystery waiting to be unlocked, and the BRAIN Initiative will change that by giving scientists the tools they need to get a dynamic picture of the brain in action and better understand how we think and how we learn and how we remember. And that knowledge could be -will be transformative". En 2014, los fondos destinados a esta investigación ascendía a 46 millones de dólares. En 2016, absorbían más de 150 millones. Uno de los resultados estrella del programa ha sido el descubrimiento de técnicas de control neural remoto del hipotálamo de ratones, mediante ondas de radio y campos magnéticos que permiten manipular sus patrones de actividad y alimentación ${ }^{17}$.

En el ámbito europeo, el Human Brain Project (HBP) es sin duda el proyecto estrella. Se trata de un consorcio internacional de investigación liderado por la Escuela Politécnica Federal de Lausana, que se financiará con fondos H2020 de la Unión Europea durante la década $2013-2023^{18}$, con fondos públicos y privados provenientes de los Estados participantes. Si el coste total previsto del proyecto es de 1.000 millones de euros, el 50 por ciento le corresponde a la Unión Europea. En él se están coordinando los esfuerzos interdisciplinares de más de 100 universidades, hospitales y centros de investigación alrededor de seis grandes tópicos: neuroinformática (generación y explotación compartida de bases de datos cerebrales), simulación cerebral (replicación de la arquitectura y la actividad cerebral en ordenadores), computación de altas prestaciones, informática médica, informática neuromórfica (desarrollo de computadores inspirados en el funcionamiento cerebral) y neurorobótica (uso de robots para ensayo de simulaciones cerebrales).

Además, el proyecto dedica una parte de los gastos elegibles al estudio de sus implicaciones éticas y sociales, en especial los riesgos que pueden generar.

De ello se encarga el Foresight Lab del King's College, liderado por el profesor Nikolas Rose $^{19}$. Los puntos clave de su evaluación de riesgos son la interacción entre hombre y máquina (automatización del trabajo, mercados basados en inteligencia artificial y, en especial la decodificación de los mecanismos de funcionamiento del cerebro y su

${ }^{17}$ Cfr. Sarah Stanley et al., "Bidirectional electromagnetic control of the hypothalamus regulates feeding and metabolism", Nature, marzo de 2016, disponible aquí

${ }^{18}$ Los proyectos-insignia financiados por la Comisión Europea en materia de tecnolgías emergentes son los cuatro siguientes: Graphene Flagship, HBP Flagship, Blue Brain Flagship y el Quantum Technology Flagship. El Blue Brain Project nace también en la Escuela Politécnica de Lausana, en 2005, y confluye con el HBP. Su propósito es crear una reconstrucción digital del cerebro mediante técnicas de ingeniería inversa aplicadas a los circuitos neuronales de los mamíferos, en especial mediante replicación de neuronas y mapeo del conectoma (los trillones de conexiones neuronales cerebrales), gracias a las posibilidades de supercomputación de altas prestaciones ofrecidas por el Blue Gene computer de IBM. En este punto, nadie duda que la revolución neurocientífica se aprovechará y retroalimentará a su vez del enorme potencial que atesora el desarollo de la informática cuántica.

19 Vid. https://www.kcl.ac.uk/sspp/departments/sshm/research/Research-Groups/BIOS/BIOSProjects/HBP/The-Human-Brain-Project.aspx 
“exportación”), y la reducción ontológica del ser humano que se puede derivar del desarrollo de una ciencia multidisciplinaria sobre el cerebro ${ }^{20}$.

De ahí que la gobernanza ética del HBP descanse sobre una estructura organizativa destinada a dar reflexividad transversal a los participante.

Sus piezas son el Ethics Advisory Board (un grupo independiente de expertos) el Ethics Rapporteur Programme (miembros de cada subproyecto científico), el Point of Registration for Ethics Concerns (PORE, portal público de acceso e información) y la Ethics Compliance (recopilación de documentacion sobre aspectos éticos de las investigaciones autorizadas). Todo el circuito (el denominado "bucle" de información y valoración) es revisado por el Researcher Awareness, desarrollado por el subproyecto de investigación Ethics and Society, que se relaciona e informa directamente a la Comisión Europea y sus evaluadores del HBP.

Aunque estemos aún lejos de "romper el código" del cerebro, la prolongación de los esfuerzos investigadores gracias a la inversión de enormes cantidades de dinero público y privado permite augurar un futuro inquietante en el que decodificar - y manipular- el cerebro desde el exterior sea no sólo posible, sino ubicuo.

Las prótesis robóticas conectadas al cerebro mediante BCI (Brain-computer interfaces) son ya una realidad. La utopía negativa de Arthur C. Clarke sobre el ordenador consciente o dotado de capacidad autónoma de aprendizaje (Hal 2000) no está ya tan lejos. No sólo los Estados Unidos y la Unión Europea están desarrollando investigación neurocientífica. También Canadá, China, Corea, Rusia o Israel lo están haciendo. El proyecto estrella japonés (Brain/MINDS Project: Brain Mapping by Integrated Neurotechnologies for Disease Studies) nos pone en alerta sobre uno de los tradicionales "carriles de aceleración" de las carreras científicas: la investigación cerebral experimental con primates (en concreto, con cerebros del mono titî) cuyas bases biológicas no son tan distintas de las humanas (y plantean menos dilemas éticos...salvo para los animalistas).

En definitiva, dado el potencial que las nuevas neurotecnologías tienen para acceder - y manipular - los mecanismos sobre los que descansan las funciones intelectuales superiores de la identidad humana, las emociones, los recuerdos, o la personalidad, y dado también el potencial de replicación artificial de tales procesos, los profesores Sara Goering y Rafael Yuste han defendido la necesidad de nuevas guías deontológicas para identificar riesgos y pautar límites éticos de las nuevas neurotecnologías ${ }^{21}$. ¿Son

\footnotetext{
${ }^{20}$ El proceso es descrito así por Christine AICARDI et at.: "from neuroscience data, neural activity is modelled in silico; from the in silico models, neuro-mimetic architectures are developed; from neuromimetic architectures, cognitive models are tailored for neuromorphic systems". Cfr. "Accompanying technology developement in the Human Brain Project: from foresight to ethics management", Futures, 2018, p. 5.

${ }^{21}$ Cfr. "On the necessity of ethical guidelines for novel neurotechnologies", Cell, vol. 167, issue 4, pp. 882-885, noviembre de 2016.
} 
protegibles los datos cerebrales? ${ }^{22}$ ¿Se pueden apropiar los mapeos cerebrales y el conectoma? ¿Será técnicamente posible "copiar" el conectoma de un individuo y conservarlo o replicarlo en una máquina o en otro cuerpo ${ }^{23}$ ?

Esto apunta hacia el lado que más nos interesa: la necesidad de identificar y proporcionar tratamiento jurídico, heterorregulación, a unos avances tecnológicos cuyas consecuencias, riesgos y límites aún no somos capaces de vislumbrar en su totalidad $^{24}$...aunque muchas creaciones literarias (Frankenstein o el moderno Prometeo) y cinematográficas distópicas (2001 Una Odisea del Espacio, Blade Runner, Yo Robot, Matrix, A.I Inteligencia Artificial) nos puedan ayudar a reflexionar sobre ello.

\section{El nacimiento del Neuroderecho ("Neurolaw")}

Es en la intersección entre esos avances tecnológicos y la regulación jurídica donde se sitúa el nacimiento de una nueva disciplina: el "neurolaw".

Podríamos traducir este término al castellano como neuroderecho o, inspirándonos en otras soluciones ya acuñadas por la doctrina, como neuronomía.

Si bien los primeros usos del término se remontan a un trabajo de perspectiva más procesalista encabezado por J. Sherrod Taylor en 1991 bajo el título "Neuropsychologists and neurolawyers" "25, la eclosión de esta disciplina puede señalarse en tres focos geográficos (EE.UU, Inglaterra y Alemania) a partir de ciertos datos que acreditan la institucionalización de un saber especializado. Repasemos sus principales aspectos.

A partir de 1985, la Dana Foundation (nacida en 1950 gracias a la labor filantrópica de Charles A. Dana y su esposa) convirtió la investigación neurocientífica en una de sus prioridades, junto con la lucha contra el cáncer. En 1992 crea la Dana Alliance for Brain Initiatives; en 2003, concede una ayuda a la American Association for the Advancement of Science, para la organización de seminarios sobre Neurociencia y Derecho destinados a formar jueces estatales y federales. Un informe de estado del arte

\footnotetext{
${ }^{22}$ Sobre el uso de los datos cerebrales y su explotación comercial, considérese el caso de Neurosky. Esta empresa ha desarrollado un sistema de monitorización en tiempo real del cerebro del conductor que es capaz de detectar la presencia de somnolencia, advirtiéndole de esta circunstancia...y dejando huella en el ordenador de abordo de tales datos, que pueden ser utilizados por las compañías aseguradoras para descargar su responsabilidad en la cobertura de los numerosos accidentes causados por somnolencia.

${ }^{23}$ Una de las esperanzas de la técnicas de criogenización que se aplican en la Alcor Life Extension Foundation consiste precisamente en evitar la muerte cerebral a la espera de avances neurocientíficos que permitan alguna suerte de replicación de su cerebro.

${ }^{24}$ Cfr. Christine AICARDI et. Al., "Accompanying technology developement in the Human Brain Project: from foresight to ethics management", Futures, 2018.

${ }^{25}$ Cfr. TAYloR, J. S., HARP, J. A., \& Elliott, T: "Neuropsychologists and neurolawyers". Neuropsychology, 1991, 5(4), pp. 293-305. El trabajo plantea las posibilidades de trabajo conjunto entre neuropsicólogos y juristas en el ámbito de las periciales judiciales de daños cerebrales traumáticos.
} 
se publica al año siguiente como consecuencia del primer seminario de $2003^{26}$. Estos seminarios han acabado organizándose también en el Reino Unido, en colaboración con la Royal Society.

En 2007, la MacArthur Foundation comenzó a financiar con 10 millones anuales el proyecto de investigación trianual Law and Neurosciences Project. A partir de ahí, el MacArthur Foundation Research Network on Law and Neurosciencie ha pasado a integrarse en la Vanderbilt University, Nashville, Tennessee, que a su vez ha impulsado un programa grado y de doctorado en la materia. En su seno, los profesores Owen D. Jones, Jeffrey D.Schall y Francis X. Shen han elaborado el volumen Law and Neuroscience (New York, Wolters Kluwer Law \& Business, 2014), primer manual sobre la materia.

El manual comienza por una introducción a los temas principales del campo, con una evaluación de dos problemas que van desde lo individual (la muerte cerebral) hasta lo colectivo (el desarrollo del cerebro del adolescente).

En la parte segunda se aborda el comportamiento y la responsabilidad desde el punto de vista neurocientífico, con las correspondientes derivas aplicativas de los nuevos descubrimientos en los tribunales de justicia.

La parte tercera analiza los fundamentos de la neurociencia cognitiva: la estructura y función cerebral, el monitoreo y manipulación del cerebro, así como sus límites y problemas.

Los tópicos presentes en la parte cuarta de este manual identifican las materias fundamentales del neuroderecho: el cerebro dañado (muerte cerebral, daños cerebrales, dolor y sufrimiento), el cerebro pensante y sintiente (memoria, emociones, detección de mentiras, capacidad de juicio), el cerebro en desarrollo y el cerebro adicto.

Finalmente, la parte quinta identifica los tres temas de futuro donde la ley está emplazada a tomar decisiones regulatorias: la potenciación de la capacidad cognitiva (por ejemplo, los nootrópicos), interfaces cerebro-máquina e inteligencia artificial.

En Inglaterra, el profesor Michael Freeman, de la University College London, ha editado los resultados de dos coloquios internacionales organizados en 2008 y 2009, bajo el título Law, Mind and Brain (Farnham, Ashgate, 2009), y Law and neurosciences (New York, Oxford University Press, 2011).

En Alemania, el profesor alemán Tade Matthias Spranger ha editado un estudio comparativo internacional de la regulación de los avances neurocientíficos bajo el título

\footnotetext{
${ }^{26}$ Cfr. Brent Garland (ed.), Neuroscience and the Law. Brain, Mind and the Scales of Justice, Nueva Yor, Dana Press y AAAS, 2004. En la primera parte de este libro (pp. 3-47), el editor se encarga de resumirnos las cuestiones planteadas en el seminario de día y medio, durante el cual se reunieron 27 especialistas (psicólogos, psiquiatras, neurocientíficos, filósofos), legisladores, jueces y profesores de derecho: monitoreo e imagen del cerebro; modificaciones del cerebro; temas legales transversales; direcciones futuras de desarrollo.
} 
International neourolaw. A comparative analysis (Berlin Heildelberg, Springer-Verlag, 2012). En su introducción, Tade Matthias afronta un estado de las variadas tecnologías disponibles de estudio del cerebro y sus implicaciones legales, para a continuación repasar comparadamente el estado de la regulación de tales implicaciones en distintos países: Australia, Austria, Brasil, Canadá, Consejo de Europa, Finlandia, Francia, Alemania, Grecia, Italia, Japón, Holanda, Suiza, Nueva Zelanda, Turquía, Inglaterra y Estados Unidos.

Los campos de análisis legal son seis: el debate sobre la responsabilidad criminal; la utilización pericial de los avances neurocientíficos (polígrafos, pruebas del dolor en asuntos de responsabilidad civil); acceso al nivel cerebral primario del consumidor (neuromarketing y neuroeconomía); BCI's (Brain-Computer interfaces); la muerte cerebral y, por último, la regulación de los estándares de investigación neurocientífica, en especial de los frecuentes hallazgos casuales ("incidental findings"27) que genera la exploración cerebral experimental. La propia concepción del libro ayuda a entender la necesidad de una discusión jurídica transnacional sobre todo ello. De ahí que también se explore la codificación de regulaciones neurocientíficas de la UNESCO y el Consejo de Europa.

La pregunta que cabe hacerse ahora es cómo traducir y tematizar todo esto en las claves del paradigma clásico de nuestro derecho administrativo. ¿Nos afectan realmente todos estos desarrollos? ¿En qué medida debemos ocuparnos de ellos? ¿Debemos entregar el estudio y regulación de estas materias a otras áreas del derecho?

\section{Un hilo de Ariadna: nuestro derecho de la investigación biomédica}

Quizá el mejor modo de afrontar una reflexión jurídica sobre la naturaleza de los problemas que la neurociencia lanza a nuestra tradición de conocimiento sea acudir a otras experiencias precedentes de regulación.

Propongo tomar la revolución genética como punto de partida más próximo para ese posible hilo conductor.

El dominio de distintas técnicas de manipulación genética durante los últimos decenios del siglo XX y el desciframiento de la secuenciación del genoma humano han permitido superar importantes fronteras científicas relativas al conocimento biológico del ser humano. Estos avances científicos sobre el dominio de las bases biológicas de la vida han provocado la necesidad de una regulación jurídica de las técnicas terapéuticas de manipulación genética disponibles, cuyos dilemas éticos y aspectos regulatorios siguen provocando amplios debates sociales -y jurídicos- a escala internacional.

\footnotetext{
${ }^{27}$ Sobre ello, vid. Susan Wolf, Jordan PARAdise y Charlisse CAGA-ANAN, "The Law of incidental findings in human subject research: establishing researcher's duties", J Law Med Ethics 36 (2), 2008, pp. 361-383, disponible aquí.
} 
Jalones de esa regulación jurídica son el Convenio para la protección de los derechos humanos y la dignidad del ser humano con respecto a las aplicaciones de la Biología y la Medicina (Convenio relativo a los derechos humanos y la biomedicina), hecho en Oviedo el 4 de abril de 1997 en el seno del Consejo de Europa, que fue ratificado por España en 1999, entrando en vigor el 1 de enero de 2000. El protocolo adicional de este Convenio, sobre trasplante de órganos y de tejidos de origen humano, hecho en Estrasburgo en 2002, ha sido ratificado por el Reino de España, entrando en vigor el 1 de abril de $2015^{28}$. España contó con una de las primeras leyes sobre reproducción asistida, la Ley 35/1988, de 22 de noviembre, sobre técnicas de reproducción asistida, hoy sustituída por la Ley 14/2006, de 26 de mayo, sobre reproducción humana asistida. En ella se incluye una cláusula abierta de definición de las técnicas disponibles, y una regulación de las técnicas de diagnóstico genético preimplantacional.

A resultas de las preocupaciones surgidas a propósito del desarrollo imparable de estas técnicas se ha promulgado en nuestro país la Ley 14/2007, de 3 de julio, de investigación biomédica.

Esta Ley articula el derecho -constitucional- a la investigación con los principios de la integridad de las personas y la protección de la dignidad e identidad del ser humano en cualquier investigación biomédica que implique intervenciones sobre seres humanos ${ }^{29}$. No es necesario recordar que desde un punto de vista constitucional la dignidad humana y el libre desarrollo de su personalidad es fundamento del orden y la paz social (art. 10.1 $\mathrm{CE}$ ), pero la libre investigación científica es igualmente un derecho fundamental (art. 20.1.b) $\mathrm{CE}^{30}$.

Estos principios son los fundamentos sobre los que se asienta la libre autonomía de la persona, a la que se anclan a su vez los derechos de otorgar consentimiento y obtener información previa sobre cualquier tratamiento o investigación médica, los derechos a no ser discriminado y el derecho a la protección de los datos de carácter personal. El artículo $2 \mathrm{f}$ ) establece que la investigación biomédica se desarrollará de acuerdo con el principio de precaución para prevenir y evitar riesgos para la vida y la salud.

\footnotetext{
${ }^{28}$ Vid. Instrumento de ratificación en $\mathrm{BOE} \mathrm{n}^{\circ} 25$, de 29 de enero de 2015.

${ }^{29}$ El artículo 1 de la ley cita explícitamente, "en particular", una serie de ámbitos de investigación biomédica: a) Las investigaciones relacionadas con la salud humana que impliquen procedimientos invasivos; b) La donación y utilización de ovocitos, espermatozoides, preembriones, embriones y fetos humanos o de sus células, tejidos u órganos con fines de investigación biomédica y sus posibles aplicaciones clínicas; c) El tratamiento de muestras biológicas; d) El almacenamiento y movimiento de muestras biológicas; e) los biobancos. También se incluye la realización de análisis genéticos y el tratamiento de datos genéticos de carácter personal. En cambio, los ensayos clínicos con medicamentos y productos sanitarios se remiten a su regulación específica, de mismo modo que las implantaciones de órganos, tejidos y células de cualquier origen se remiten a la Ley 30/1979, de 27 de octubre, sobre extracción y trasplante de órganos.

${ }^{30}$ No cabe sino remitirse aquí a la ponencia de Mercé DARNACULLETA recientemente presentada al congreso anual de la AEPDA en Salamanca bajo el título "Libertad de investigación científica y promoción de la ciencia en beneficio del interés general", en curso de publicación.
} 
Estos enfoques regulatorios, a mi juicio, son perfectamente extensibles a las investigaciones neurocientíficas y sus técnicas conexas, cuyo potencial de afección al libre desarrollo de la personalidad no es necesario enfatizar más.

Sin embargo, la ley 14/2007 parte de un ámbito objetivo de aplicación que no es suficientemente amplio para incluir todos los hallazgos neurocientíficos. Justamente, una de las características mas importantes del actual conocimiento neurocientífico es el hecho de ser el resultado de una confluencia de tecnologías a gran escala.

Es decir, de una aplicación a gran escala -a una escala transnacional- de una convergencia a su vez transdisciplinaria, creadora de nuevos saberes y técnicas sobre un objeto concreto, el cerebro humano, susceptible de generar múltiples aplicaciones comerciales, militares, sociales....Si esta hipótesis está correctamente formulada, su consecuencia inmediata es la necesidad de un marco global de regulación jurídica que responda adecuadamente tanto a la naturaleza transnacional del espacio como al contenido transdisciplinar del objeto a regular.

\section{El espacio regulador global}

Siguiendo los precedentes del Convenio de Oviedo de 1997, un esquema plausible podría ser el de la celebración de una convención internacional y, a partir de ahí, el despliegue de regulaciones legales internas.

La propia Unesco aprobó el 11 de noviembre de 1997 la Declaración Universal sobre el Genoma Humano y los Derechos Humanos, asimilando el genoma, al menos simbólicamente, al régimen de los bienes comunes o patrimonio de la Humanidad ${ }^{31}$. Posteriormente, la Unesco adoptó, el 16 de octubre de 2003, la Declaración Internacional sobre los datos genéticos humanos, cuyo contenido es mucho más preciso y operativo.

En 19 de octubre de 2005 se aprobaba la Declaración Universal sobre Bioética y Derechos Humanos, que aborda las cuestiones éticas relacionadas con la medicina, las ciencias de la vida y las tecnologías conexas aplicadas a los seres humanos, teniendo en cuenta sus dimensiones sociales, jurídicas y ambientales: dignidad y derechos humanos, valoración de los beneficios y efectos nocivos, autonomía y responsabilidad individual,

${ }^{31}$ El artículo 1 dice exactamente que "en sentido simbólico, el genoma humano es el patrimonio de la Humanidad". Pero esta noción general ha dejado de ser simbólica para adoptar ya un significado jurídico vinculante. Piénsese en el precedente del Tratado de las Naciones Unidas sobre los principios que deben regir las actividades de los Estados en la exploración y utilización del espacio ultraterrestre, incluso la Luna y otros cuerpos celestes sobre el espacio ultraterrestre, aprobado en diciembre de 1966 y en vigor desde el 10 de octubre de 1967. Su artículo segundo establece que el espacio ultraterrestre no puede ser objeto de apropiación nacional por reivindicación de soberanía, uso u ocupación, ni de ninguna otra manera. Y en su artículo primero fija la regla de que la exploración y utilización del espacio ultraterrestre debe hacerse en provecho e interés de todos los países, e incumben a toda la humanidad. Vid. sobre ello José Luís GORDILlo (coord.), La protección de los bienes comunes de la Humanidad. Un desafío para la politica y el derecho del siglo XXI, Madrid, Trotta, 2006. La bibliografía sobre los comunes se ha expandido desde entonces hasta hoy en un movimiento internacional back to Commons. 
consentimiento de la persona interesada, respeto de la vulnerabilidad humana, privacidad y confidencialidad, no discriminación, solidaridad y cooperación internacional, aprovechamiento compartido de los beneficios, protección de las generaciones futuras ... Sin embargo, estos instrumentos no han abandonado el status del soft law ${ }^{32}$. No se ha dado el paso de crear una convención internacional plenamente eficaz en el ámbito interno de los Estados.

Para paliar esta ausencia regulatoria, Caroline Rödiger defendió en 2012 la oportunidad de elaborar un protocolo adicional al Convenio de Oviedo, centrado en la investigación neurocientífica ${ }^{33}$. Este protocolo adicional europeo podría suponer el preludio o primer paso para una regulación mundial en el seno de la ONU.

Pese a estos esfuerzos, lo cierto es que a día de hoy el ámbito regulador no ha alcanzado aún ese estadio. Estamos en un momento en el que las regulaciones adoptan una geometría variable y desigual, que aproximan respuestas poco sistemáticas a concretos problemas.

Apenas algún país europeo ha incorporado ya alguna regulación parcial. Es el caso de Francia, en cuya revisión de 2011 de las leyes sobre bioética (Ley n 2011-814, de 7 de julio), se ha introducido un artículo 16-14 en el Código Civil, relativo a la utilizacion de la imagen cerebral: "Les techniques d'imagerie cérébrale ne peuvent être employées qu'à des fins médicales ou de recherche scientifique, ou dans le cadre d'expertises judiciaires. Le consentement exprès de la personne doit être recueilli par écrit préalablement à la réalisation de l'examen, après qu'elle a été dûment informée de sa nature et de sa finalité. Le consentement mentionne la finalité de l'examen. Il est révocable sans forme et à tout moment". Se trata de una extensión a las técnicas diagnósticas neurales de dos reglas inicialmente previstas para la genética (prestación escrita y previa de consentimiento informado, y derecho a conocer su naturaleza y finalidad), con las miras puestas en la válida utilización de imágenes cerebrales en informes periciales judiciales.

Más allá de estas soluciones nacionales, la replicación robótica de la inteligencia humana, es decir, la posibilidad de construir máquinas que repliquen mediante técnicas de inteligencia artificial el funcionamiento del cerebro humano (brain-inspired computing) es el campo en el que se ha emanado la Resolución del Parlamento Europeo, de 16 de febrero de 2017, con recomendaciones destinadas a la Comisión sobre normas de Derecho civil sobre robótica ${ }^{34}$.

La resolución se centra en las complejas cuestiones relativas a la responsabilidad civil por daños ocasionados por robots; pero incorpora también una "Carta sobre robótica",

\footnotetext{
${ }^{32}$ Cfr. Darryl MACER, "Neurolaw and UNESCO bioethics Declarations", en Tade Matthias SPRANGER, International Neurolaw. A Comparative analysis, op. cit., p. 330.

${ }^{33}$ Cfr. "The Council of Europe's next "Additional Protocol on Neuroscientific Research"?, en Tade Matthias SPRANGER (ed.), International Neurolaw, op. cit. pp. 103-115.

${ }^{34}$ La Resolución se basa sobre el trabajo prospectivo de STOA, la oficina del Parlamento Europeo de evaluación de las opciones científicas y tecnológicas (Science and Technology Options Asessment)
} 
que propone: (a) un código ético de conducta para los ingenieros en robótica; (b) un código deontológico para los comités de ética de la investigación; (c) una licencia para los diseñadores y (d) una licencia para los usuarios.

A la postre, la creciente autonomía de los robots suscita la cuestión de su naturaleza y de si pertenecen a una de las categorías jurídicas existentes o si debe crearse una nueva categoría con sus propias características jurídicas. Ni que decir tiene que la Resolución emplaza a la Comisión a adoptar una Directiva que proporcione definiciones y reglas con amplia repercusión en materia de responsabilidad patrimonial de las Administraciones Públicas, al amparo del artículo 114 TFUE.

Estamos ante el embrión jurídico destinado a canalizar la regulación de la revolución industrial que se está produciendo en el campo de la tecnología robótica y el desarrollo de la inteligencia artificial, uno de cuyas consecuencias es la capacidad de autoaprendizaje y decisión autónoma ${ }^{35}$. No por casualidad, la Resolución incorpora como principios las denominadas leyes de la robótica de Asimov, acuñadas en su novela Runaround de 1941. También se incorpora una referencia a la salvaguarda de los datos de carácter personal que afectan a la intimidad de las personas, y que pueden ser generados por máquinas mediante el cruce de big data y el uso de algoritmos avanzados sin el debido control humano. La Resolución, en efecto, cita expresamente en este sentido el Reglamento (UE) 2016/679 del Parlamento Europeo y del Consejo, de 27 de abril de 2016, relativo a la protección de las personas físicas en lo que respecta al tratamiento de datos personales y a la libre circulación de estos datos y por el que se deroga la Directiva 95/46/CE (Reglamento general de protección de datos). Otro desafío planteado por la Resolución del Parlamento Europeo es la necesidad de un enfoque normativo adecuado y coherente a escala de la Unión para que la industria europea pueda desarrollar sus inversiones en este sector con seguridad jurídica, sin que se produzca una deslocalización tecnológica a terceros países.

Todo ello pone de manifiesto la necesaria globalización de una respuesta jurídica a los continuos avances tecnológicos derivados del cruce sistemático de saberes científicos. En particular, los derivados de la investigación neurocientífica y su uso: si el objeto pertenece a la propia especie humana, el espacio regulador debería ser global.

Ahora bien, esto no significa que la reflexión sobre las consecuencias regulatorias de los avances neurocientíficos deba quedar al margen del derecho interno. Al contrario, pienso que van a surgir muchos campos en los que el Derecho administrativo resultará interpelado de maneras que todavía no podemos conjeturar.

En este sentido, me parece oportuno plantear algunas hipótesis sobre un concreto campo positivo de aplicación de todo lo discutido. De esta manera, intentando "rasear el balón", espero que se pueda valorar mejor las tareas que tenemos por delante.

\footnotetext{
${ }^{35}$ Sobre el impacto de la inteligencia artificial en las profesiones jurídicas, Julián VALERO TORRIJOS ha organizado el 11 de abril de 2018 una muy interesante jornada de debate, cuyo vídeo puede verse aquí.
} 


\section{Una coda final de vuelta a casa: neurociencia y cuantificación del daño moral en procesos de responsabilidad patrimonial}

La cuantificación y prueba del dolor - recte: de su percepción por el cerebro- mediante marcadores neurológicos posee una gran potencialidad como instrumento terapéutico y de pericia técnica.

En concreto, sus posibles repercusiones en los procedimientos de reclamación por responsabilidad patrimonial, incluída la administrativa, son enormes: no sólo para acreditar el daño (físico y psíquico) causado por lesiones, sino también para evaluar de manera más ajustada al caso concreto, al individuo concreto, la intensidad -e indemnizabilidad- del denominado "daño moral", cuya determinación u objetivación se realiza en la actualidad en nuestro ordenamiento mediante la técnica de la presunción: se presume que el dolor físico y sus secuelas llevan aparejado un daño o dolor moral complementario. Sobre esta presunción, y una vez cuantificado pericialmente el daño físico producido, se fija una horquilla ciertamente amplia para el pretium doloris, por referencia a la tasación del daño físico con arreglo a tablas o baremos ${ }^{36}$.

\section{INDEMNIZACIONES POR SECUELAS (TABLA 2.B)}

Perjuicio personal particular

\section{PERJUICIOS PARTICULARES}

\section{Daños morales complementarios por perjuicio psicofisico}

Cuando una sola secuela alcanza al menos 60 puntos o el resultado de las concurrentes alcanza al menos 80 puntos.

De $19.200 €$ hasta $96.000 €$

\section{Daños morales complementarios por perjuicio estético}

Cuando alcanza al menos 36 puntos.

De $9.600 €$ hasta $48.000 €$

\section{Perjuicio moral por pérdida de calidad de vida ocasionada por las secuelas}

Muy Grave

De $90.000 €$ hasta $150.000 €$

${ }^{36}$ El sistema actual trae causa de la Ley 35/2015, de 22 de septiembre, de reforma del sistema para la valoración de los daños y perjuicios causados a las personas en accidentes de circulación, que ha introducido un nuevo título IV en el Real Decreto Legislativo 8/2004, de 29 de octubre, por el que se aprueba el texto refundido de la Ley sobre responsabilidad civil y seguro en la circulación de vehículos a motor. El artículo 33. 3 del RDL 8/2004 dice ahora que "El principio de la reparación íntegra rige no sólo las consecuencias patrimoniales del daño corporal sino también las morales o extrapatrimoniales e implica en este caso compensar, mediante cuantías socialmente suficientes y razonables que respeten la dignidad de las víctimas, todo perjuicio relevante de acuerdo con su intensidad". De ahí la necesidad de presentar informes médicos que acrediten y permitan valorar las secuelas y sus consecuencias personales. La regulación de los daños morales complementarios por perjuicios psicofísicos, orgánicos, sensoriales, estéticos y por pérdida de calidad de vida se contiene en los artículos 105 a 110. 


\begin{tabular}{|l|c|}
\hline Grave & $\begin{array}{c}\text { De } 40.000 € \text { hasta } \\
100.000 €\end{array}$ \\
\hline Moderado & $\begin{array}{c}\text { De } 10.000 € \text { hasta } \\
50.000 €\end{array}$ \\
\hline Leve & $\begin{array}{c}\text { De } 1.500 € \text { hasta } \\
15.000 €\end{array}$ \\
\hline $\begin{array}{l}\text { 4. Perjuicio moral por pérdida de calidad de vida de los } \\
\text { familiares de grandes lesionados }\end{array}$ & $\begin{array}{c}\text { De } 30.000 € \text { hasta } \\
145.000 €\end{array}$ \\
\hline 5. Pérdida de feto a consecuencia del accidente & \begin{tabular}{l}
$15.000 €$ \\
\hline Si la pérdida tuvo lugar en las primeras 12 semanas de gestación
\end{tabular} \\
\hline Si la pérdida tuvo lugar a partir de las 12 semanas de gestación & $30.000 €$ \\
\hline 6. Perjuicio Excepcional & Hasta $25 \%$ \\
\hline
\end{tabular}

Baremo según la Ley 35/2015, de 22 de septiembre

Pero dentro de esa horquilla, la discrecionalidad del juzgador es amplia, y en consecuencia, amplia es la jurisprudencia ${ }^{37}$. Aunque se acuda en algunas ocasiones a pericias psicológicas (entrevistas, etc.), el daño moral sigue siendo pasto de decisiones discrecionales, de arbitrio judicial "sobre el valor del padecimiento humano en las distintas situaciones de la vida" ${ }^{38}$.

Una de las novedades de la Ley 35/2015 ha sido la previsión de una vía extrajudicial de resolución de conflictos entre perjudicados y aseguradoras. Dentro de esa vía, se permite ahora que se soliciten informes periciales especializados sobre el daño "corporal" derivado de accidentes de circulación a los Institutos de Medicina Legal y Ciencias Forenses.

Esta prestación de servicios administrativos se regula con detalle en el Real Decreto 1148/2015, de 18 de diciembre, por el que se regula la realización de pericias a solicitud de particulares por los Institutos de Medicina Legal y Ciencias Forenses, en las reclamaciones extrajudiciales por hechos relativos a la circulación de vehículos a motor. En concreto, en su anexo se recoge el modelo del informe forense sobre prevision/valoracion de daños personales. Como uno de los contenidos obligatorios de la pericia están los siguientes:

${ }^{37}$ Cfr. Joan Picó I Junoy, "La prueba del dolor", en Michele TARufFo y Jordi NiEva Fenoll, Neurociencia y proceso judicial, op. cit. Pp. 83-95; Cristóbal Molina NAVARReTe, "El "precio" del "dolor moral" del funcionario. ¿Por qué es menor en el orden contencioso-administrativo? (Comentario a la Sentencia del Tribunal Superior de Justicia de Castilla y León/Valladolid de 14 de noviembre de 2014, rec. núm. 1684/2011)", Estudios Financieros. Revista de trabajo y seguridad social, no 383, 2015, pp. 207-212. De especial utilidad en este ámbito es el trabajo de Gabriel DomÉnECH PASCUAL, "La cuantificacion de los daños morales causados por las Administraciones Públicas", en Fernando GómEZ POMAR e Ignacio MARÍN GARCía (coords.) El daño moral y su cuantificación, Barcelona, Bosch- Wolters Kuwer, 2017, pp. 519-558.

${ }^{38}$ Cfr. STS (sala $3^{\text {a }}$ ) de 5 de mayo de 2009, RJ 5167. 
13.- Daño moral complementario por perjuicio psico-físico:

SI

NO

14.- Daño moral complementario por perjuicio estético:

SI

NO

\section{5.- Perjuicio moral por pérdida de calidad de vida de familiares:}

SI NO

¿Será posible en un futuro próximo cuantificar científicamente el daño moral, más allá de una simple respuesta binaria no motivada objetivamente?

Si ya se han desarrollado tecnologías para medir el dolor físico en pacientes que no pueden comunicarlo (neonatos, pacientes con deterioro cognitivo, entubados, anestesiados, etc.), no parece descabellado pensar en la aplicación de técnicas de medición científica de las "huellas" cerebrales del sufrimiento moral o psíquico, dada la certidumbre que tal valoración puede arrojar sobre procesos cuyo coste económico agregado es indudable.

\section{Bibliografía}

Christine AICARDI et at.: "Accompanying technology developement in the Human Brain Project: from foresight to ethics management", Futures, 2018.

Mercé DARNACULLETA: "Libertad de investigación científica y promoción de la ciencia en beneficio del interés general", ponencia presentada al XIII Congreso de la Asociación Española de Profesores de Derecho Administrativo, Salamanca 9-10 de febrero de 2018, en curso de publicación.

Eduardo DEMETRIO CRESPO: Neurociencias y Derecho Penal, Buenos AiresMontevideo, editorial B de F, 2017.

Eduardo Demetrio CRESPO (dir.) y Manuel MAROTO CALATAYUd (coord.): Neurociencias y derecho penal: nuevas perspectivas en el ámbito de la culpabilidad y tratamiento jurídico-penal de la peligrosidad, Madrid, Edisofer, 2013.

Gabriel DOMÉNECH PASCUAL: "La cuantificacion de los daños morales causados por las Administraciones Públicas", en Fernando GóMEZ POMAR e Ignacio MARÍN GARCÍA (coords.) El daño moral y su cuantificación, Barcelona, Bosch- Wolters Kuwer, 2017.

Nita FARAHANY: "3 reasons brain science is terrific and terrifying", World Economic Forum, 21 enero de 2016.

Bernardo José FEIJOO SÁNCHEZ: Derecho Penal de la culpabilidad y neurociencias, Civitas Thomson Reuters, 2012.

Michael FreEmAn: Law, Mind and Brain, Farnham, Ashgate, 2009. 
Law and neurosciences, New York, Oxford University Press, 2011.

Brent Garland (ed.): Neuroscience and the Law. Brain, Mind and the Scales of Justice, Nueva Yor, Dana Press y AAAS, 2004.

José Luís GoRDILlo (coord.): La protección de los bienes comunes de la Humanidad. Un desafío para la política y el derecho del siglo XXI, Madrid, Trotta, 2006.

Owen D. Jones, Jeffrey D.Schall y Francis X. SHEN: Law and Neuroscience, New York, Wolters Kluwer Law \& Business, 2014.

Darryl MACER: "Neurolaw and UNESCO bioethics Declarations", en Tade Matthias SPRANGER, International Neurolaw. A Comparative analysis, Berlin-Heildelberg, Springer-Verlag, 2012.

Cristóbal Molina NAVARRETE: "El "precio" del "dolor moral" del funcionario. ¿Por qué es menor en el orden contencioso-administrativo? (Comentario a la Sentencia del Tribunal Superior de Justicia de Castilla y León/Valladolid de 14 de noviembre de 2014, rec. núm. 1684/2011)", Estudios Financieros. Revista de trabajo y seguridad social, $\mathrm{n}^{\mathrm{o}} 383,2015$.

Joan PICÓ I JUNOY: "La prueba del dolor", en Michele TARUFFO y Jordi NIEVA FENOLL (dirs.), Neurociencia y proceso judicial, Madrid, Marcial Pons, 2013.

Juli PONCE SOLÉ: "Nudging, simplificación procedimental y buen gobierno regulatorio. El Derecho Administrativo del siglo XXI y sus relaciones con las ciencias sociales", en Luis Míguez Macho, Marcos Almeida Cerreda y Diana SAntiago IGlesias, La simplificación de los procedimientos administrativos: actas del IX Congreso de la Asociación Española de Profesores de Derecho Administrativo, Santiago de Compostela, EGAP, 2014.

Juli Ponce Solé y Agustí Cerrillo Martínez: Innovación en el ámbito del buen gobierno regulatorio: ciencias del comportamiento, transparencia y prevención de la corrupción, INAP, Madrid, 2017.

José Antonio RAmos VÁZQUeZ: Ciencia, libertad y Derecho penal (aporías del determinismo y defensa de la libertad de acción como base del sistema penal, Valencia, Tirant Lo Blanch, 2013.

Jesús María Silva SÁNCHEZ: "El retorno de la inocuización. El caso de las reacciones jurídico penales frente a los delincuentes sexuales violentos", en Luis Alberto ARROYO ZAPATERo e Ignacio BERdugo GómeZ DE LA TORRE (coords.). Homenaje al Doctor Marino Barbero Santos: in memoriam, Ediciones de la Universidad de Salamanca, Cuenca, 2001.

Tade Matthias SPRANGER (ed.): International neurolaw. A comparative analysis, BerlinHeildelberg, Springer-Verlag, 2012.

Caroline RöDIGER: "The Council of Europe's next "Additional Protocol on Neuroscientific Research"?, en Tade Matthias SPRANGER (ed.), International Neurolaw. A comparative analysis, Berlin-Heildelberg, Springer-Verlag, 2012. 
C. Summerfield y A. Blangero: "Perceptual decision-making: what do we know and what do we not know", en Jean-Claude DreHER y Léon TREMBlay (eds.), Decision Neuroscience, Londres, Academic Press, 2017.

Cass SunStEIn: Paternalismo libertario, Barcelona, Herder, 2017.

The ethics of influence. Government in the age of behavioral sciences, Cambridge University Press, 2016.

Sarah STANLEY et al.: "Bidirectional electromagnetic control of the hypothalamus regulates feeding and metabolism", Nature, marzo de 2016.

Michele TARUfFo y Jordi NiEva FenOll (dirs.): Neurociencia y proceso judicial, Madrid, Marcial Pons, 2013.

John Sherrod TAYLOR, J. Anderson HARP \& Tyron ElliOTT: "Neuropsychologists and neurolawyers". Neuropsychology, 1991, 5(4).

Richard Thaler y Cass Sunstein: Un pequeño empujón (nugdge), Madrid, Taurus, 2009.

G. UGAZIO y C. RUFF: "Neural control of social decisions: causal evidence from brain stimulation studies", en Jean-Claude DreHER y Léon Tremblay (eds.), Decision Neuroscience, Londres, Academic Press, 2017.

Susan Wolf, Jordan PARAdise y Charlisse CAGA-ANAN, "The Law of incidental findings in human subject research: establishing researcher's duties", J Law Med Ethics 36 (2), 2008. 